\title{
High expression of the secreted protein dickkopf homolog 4: Roles in invasion and metastasis of renal cell carcinoma and its association with Von Hippel-Lindau gene
}

\author{
WEI ZHAI，GUANG-HUI HU，JUN-HUA ZHENG，BO PENG，MIN LIU，JIAN-HUA HUANG, \\ GUANG-CHUN WANG, XU-DONG YAO and YUN-FEI XU \\ Department of Urology, Shanghai Tenth People's Hospital, \\ Tongji University School of Medicine, Shanghai 200072, P.R. China
}

Received September 22, 2013; Accepted December 23, 2013

DOI: $10.3892 /$ ijmm.2014.1673

\begin{abstract}
The aim of this study was to investigate the effects of the dickkopf homolog 4 (DKK4)/Wnt/ $\beta$-catenin signaling pathway on tumorigenesis and metastasis in clear cell renal cell carcinoma (ccRCC), as well as to elucidate the underlying mechanisms. We examined the expression of DKK4 in 30 cases of ccRCC and matched adjacent normal tissues, and investigated its correlation with clinicopathological characteristics. Stable DKK4-transfected cells were established, and DKK4 functional analyses were performed, including a T-cell factor/lymphoid enhancer factor (TCF/LEF) reporter assay, and experiments on cell viability, apoptosis, invasive capability and tumor growth in vivo. Finally, western blot analysis was performed to detect Von Hippel-Lindau (VHL) expression in 50 clinical specimens. The expression levels of the DKK4, $\beta$-catenin and $\beta$-catenin downstream target genes, cyclin D1 and c-myc, were determined in the these specimens, as well as in RCC4(-), T3-14(+) cell lines by qRT-PCR and western blot analysis. The same tests were also performed in human embryonic kidney (HEK)293 cells which were transfected with the pCDH-DKK4 plasmid. After 6 weeks the tumor weight significantly increased in the mice transfected with the tumor cells. DKK4 mRNA and protein expression levels were significantly upregulated $(\mathrm{p}<0.001)$. DKK4 was distinctly overexpressed $(68.0 \%)$ in all patient tissues. VHL(-) samples accounted for $60.0 \%$ of all samples, while DKK4 expression was significantly upregulated in $50 \%$ of these samples, indicating a correlation with VHL(-) expression $(\mathrm{r}=0.403, \mathrm{p}<0.05)$. We also observed reduced expression levels
\end{abstract}

Correspondence to: Dr Yun-Fei Xu or Dr Jun-Hua Zheng, Department of Urology, Shanghai Tenth People's Hospital, Tongji University School of Medicine, No. 301 Yanchang Road, Shanghai 200072, P.R. China

E-mail: yunfeixu22@hotmail.com

E-mail: jacky_zw2002@hotmail.com

Key words: dickkopf homolog 4, invasion, metastasis, clear cell renal cell carcinoma, von Hippel-Lindau, Wnt/ $\beta$-catenin signaling pathway of cyclin D1, c-myc and $\beta$-catenin (to a greater extent) in the VHL(-), RCC4(-) and T3-14(+) cells, as well as in the stably transfected HEK293 cells. DKK4 may be an oncogene, and its upregulated expression may be involved in the pathogenesis of ccRCC as a downstream gene of VHL. By activating other pathways apart from the $\mathrm{Wnt} / \beta$-catenin pathway, DKK4 may play an important role in ccRCC tumorigenesis and metastasis.

\section{Introduction}

Renal cell carcinoma ( $\mathrm{RCC}$ ) is the most common renal parenchyma tumor, accounting for approximately $3 \%$ of adult malignant tumors, presenting the highest morbidity and mortality rate in tumors of the urinary system, second to bladder cancer (1). Clear cell RCC (ccRCC) represents $>75 \%$ of all RCC cases and is the most aggressive of all known RCC subtypes, presenting a complex biological behavior which differs significantly between individuals (2). The post-operative recurrence rate of ccRCC reaches up to $20-40 \%(3,4)$. Once metastatic disease develops, the 5-year survival rate for patients with ccRCC decreases from $60 \%$ to $<5 \%$ (5). Thus, it is mandatory to elucidate the mechanisms involved in the pathogenesis of RCC in order to discover novel therapeutic targets.

Wnts are secreted signaling proteins that influence multiple processes ranging from stem cell control to cell differentiation and polarity (6). The Wnt signaling pathway is involved in the occurrence and development of a variety of carcinomas, such as hepatocellular (7) and ovarian cancer (8), melanoma (9), medulloblastoma (10) and RCC (11). The Wnt/ $\beta$-catenin signaling pathway is also known as the canonical Wnt pathway. Genetic and embryological studies have revealed that $\beta$-catenin is a component of the Wnt signaling pathway and that it exhibits signaling functions $(12,13)$. When $\beta$-catenin accumulates, it can combine with the transcription factor, T-cell factor/lymphoid enhancer factor (TCF/LEF), in the nucleus, and then activate the transcription of the signaling target gene of Wnt (14). Dickkopf (DKK) family proteins are among $5 \mathrm{Wnt}$ antagonist families, with DKK1-DKK4 being the 4 main members (15). DKK4 blocks signal transduction by binding to the Wnt co-receptor (16). As a negative feedback regulator, DKK4 plays a crucial role in regulating the $\mathrm{Wnt} / \beta$-catenin signaling 
pathway; it inhibits the proliferation and invasion of colon cancer cells by blocking the canonical Wnt pathway (17). DKK4 inhibits the TCF/LEF transcription complex, and regulates the Wnt/ $\beta$-catenin pathway (18). It has been proven that the canonical Wnt pathway is activated in ccRCC tissues, and that patients with high $\beta$-catenin expression levels have bad prognosis $(19,20)$. However, there are limited studies on the effect of DKK4 on the proliferation and invasion of ccRCC cells.

Germ line mutations of the Von Hippel-Lindau (VHL) gene lead to the development of a variety of tumors, including ccRCC. An early event during the evolution of ccRCC is the loss of function of the VHL gene (21). Gene deletion, mutation and methylation are common causes of abnormal VHL expression, and approximately $50 \%$ of all ccRCC cases present with these abnormalities (22). The VHL gene functions in several pathways associated with carcinogenesis, most notably the hypoxia-inducible pathway (23). The abnormal expression of VHL induces hypoxic conditions and activates the hypoxiainducible factor (HIF), a transcription factor. Thus, VHL participates in a number of pathways, including $\beta$-cateninmediated signaling pathways (19).

Considering the fact that both VHL and DKK4 function as inhibitors of the Wnt/ $\beta$-catenin signaling pathway, in the current study, we thus aimed to investigated the interaction between DKK4 and VHL and the Wnt/ $\beta$-catenin pathway in ccRCC. We also examined the expression of DKK4 in human renal cell lines and tumor tissues. In addition, stable DKK4transfected cell lines were established in order to examine the effects of DKK4 on cell proliferation and metastasis in vitro. Finally, we investigated the expression of DKK4 to determine its clinical relevance in the prognosis of 30 patients with RCC in order to further elucidate the role of DKK4 in ccRCC.

\section{Materials and methods}

Clinical samples. A total of 30 patients (18 males vs 12 females; average age, $59 \pm 7.8$ years) with pathologically confirmed ccRCC were enrolled in this study (Tenth Peoples' Hospital of Tongji University, Shanghai, China). The patients were classified according to the Fuhrman nuclear grading system and were staged according to the tumor-lymph nodemetastasis (TNM) classification system (Table I), in which T refers to the size of the original (primary) tumor and whether it has invaded nearby tissue, $\mathrm{N}$ refers to the degree of spread to regional lymph nodes, and $\mathrm{M}$ refers to distant metastasis (spread of cancer from one part of the body to another). All patients had not undergone any prior radiotherapy, chemotherapy or immunotherapy. Samples were obtained from tumor and adjacent normal kidney tissues from the patients with ccRCC after obtaining written informed consent. The study was approved by the Ethics Committee of the Tenth Peoples' Hospital of Tongji University.

Cell culture. The RCC cell lines, A-498 and 786-O, as well as RCC4(-) and T3-14(+), were purchased from the Cell Institute, Shanghai Branch of Chinese Academy of Sciences. The cell lines were cultured in $50 \mathrm{ml}$ Roswell Park Memorial Institute (RPMI)-1640 medium (Gibco-BRL, Carlsbad, CA, USA) and supplemented with $10 \%$ fetal bovine serum (FBS) (HyClone, Logan, UT, USA) at $37^{\circ} \mathrm{C}$ with $5 \% \mathrm{CO}_{2}$.
Plasmid construction and DNA transfection. After having constructed pCDH-DKK4, a plasmid that contained the human full-length combinational DNA fragment of DKK4, the 786-O and A-498 cells were transfected with it, using the GeneAmp PCR System 9700 (Perkin-Elmer, Waltham, MA, USA) in order to create stable cell lines that overexpressed DKK4. Transfected cells were selected by culturing them with G418 $(150 \mu \mathrm{g} / \mathrm{ml})$ for 2 months. Empty vector transfectants were used as controls. Single colonies of stable transfectants were isolated for further analysis based on their DKK4 expression levels. The top 2 stable clones of 786-O cells that had the highest DKK4 mRNA expression levels compared to those of the controls were selected, and named DKK4 clones I and II, respectively. DKK4 expression levels in the cells were confirmed by quantitative reverse transcriptase-polymerase chain reaction (qRT-PCR) before carrying out the following experiments. All experiments were performed in triplicate.

Cell viability assay. Stably transfected 786-O cells with high expression levels of DKK4 mRNA were maintained for 1 week in medium supplemented with $150 \mu \mathrm{g} / \mathrm{ml} \mathrm{G} 418$. One week later, cell viability was detected by MTS assay (GMS10043 V.A, Genmed Scientifics, Inc., Arlington, MA, USA) according to the manufacturer's instructions. Trypan blue $(0.04 \%$, Sigma, St. Louis, MO, USA) exclusion was also used to identify viable cells with intact plasma membranes. To verify the effect of DKK4 on renal cancer cells, we also performed similar MTS assays using the A-498 cell line.

Cell invasion assay. The invasion ability of the stably transfected 786-O and A-498 cells with high expression levels of DKK4 was examined by Transwell assay (Corning, Inc., Lowell, MA, USA). Transwell chambers (Corning) were used according to the manufacturer's instructions. In brief, the infected cells were harvested, resuspended in serum-free medium and subsequently transferred to hydrated Matrigel chambers $(50 \mu \mathrm{l} /$ well). The chambers were then incubated for $48 \mathrm{~h}$ in culture medium which was added to the lower chambers prior to examination. The cells on the upper surface of the filter were scraped and washed away, while the invaded cells on the lower surface were fixed and stained with $0.04 \%$ trypan blue for $2 \mathrm{~h}$. Finally, the invaded cells were counted under a microscope and the relative number was thus calculated.

Apoptosis assay. Stably transfected DKK4 clone I and clone II cells, as well as the control cells were washed twice in phosphate-buffered saline (PBS) and their density was adjusted to $1 \times 10^{6} / \mathrm{ml}$. The cells were stained in the dark for $15 \mathrm{~min}$ with $5 \mu \mathrm{l}$ of Annexin $\mathrm{V}$ and $5 \mu \mathrm{l}$ of propidium iodide (PI) (Invitrogen Life Technologies, Carlsbad, CA, USA) at room temperature, according to the manufacturer's instructions (Biosea, Beijing, China). The apoptotic cells were detected and measured on a flow cytometer (Perkin-Elmer) and examined under an inverted fluorescence microscope (ChongQing Optical \& Electrical Instrument Co., ChongQing, China).

TCF/LEF. The TCF/LEF assay with TCF-reporter plasmids (TOPFlash, which contains a wild-type TCF binding site; and FOPFlash, which contains a mutant TCF binding site; Millipore, Billerica, MA, USA) were used to monitor the 
Table I. Correlation between dickkofp-4 (DKK4) expression and clinicopathological parameters $(n=30)$.

\begin{tabular}{|c|c|c|c|}
\hline \multirow[b]{2}{*}{ Parameters } & \multicolumn{3}{|c|}{ No. of patients (\%) } \\
\hline & $\begin{array}{l}\text { High } \\
\text { expression } \\
(\mathrm{n}=19)\end{array}$ & $\begin{array}{c}\text { Low } \\
\text { expression } \\
(n=11)\end{array}$ & P-value \\
\hline \multicolumn{4}{|l|}{ Age (years) } \\
\hline Mean \pm SD & $64 \pm 8.3$ & $59 \pm 7.8$ & $0.047^{\mathrm{a}}$ \\
\hline \multicolumn{4}{|l|}{ Gender } \\
\hline Male, $n=18$ & $10(55.6)$ & $8(45.4)$ & \\
\hline Female, $n=12$ & $9(75.0)$ & $3(25.0)$ & 0.25 \\
\hline \multicolumn{4}{|l|}{ Fuhrman grade } \\
\hline $1, \mathrm{n}=7$ & $6(85.7)$ & $1(24.3)$ & Reference \\
\hline $2, n=20$ & $11(55.0)$ & $9(45.0)$ & 0.15 \\
\hline $3, n=3$ & $2(66.7)$ & $1(33.3)$ & 0.55 \\
\hline \multicolumn{4}{|c|}{ Pathological stage } \\
\hline $\mathrm{I}, \mathrm{n}=5$ & $4(80.0)$ & $1(20.0)$ & \\
\hline II, $\mathrm{n}=20$ & $11(55.0)$ & $9(45.0)$ & \\
\hline $\mathrm{I}+\mathrm{II}, \mathrm{n}=25$ & $15(60)$ & $10(40)$ & Reference \\
\hline III, $n=2$ & $1(50)$ & $1(50)$ & \\
\hline $\mathrm{IV}, \mathrm{n}=3$ & $2(66.7)$ & $1(33.3)$ & \\
\hline $\mathrm{III}+\mathrm{IV}, \mathrm{n}=5$ & $3(60.0)$ & $2(40.0)$ & 1.00 \\
\hline
\end{tabular}

Pathological tumor

classification

$\begin{array}{lll}\text { pT1 }, \mathrm{n}=15 & 10(66.7) & 5(33.3) \\ \text { pT2, } \mathrm{n}=10 & 4(40.0) & 6(60.0) \\ \text { pT1 }+\mathrm{pT} 2, \mathrm{n}=25 & 14(56.0) & 11(44.0) \\ \text { pT3, n=4 } & 3(75.0) & 1(25.0) \\ \text { pT4, n=1 } & 1(100.0) & 0(0.0) \\ \text { pT3 }+ \text { pT4, n=5 } & 4(80.0) & 1(20.0)\end{array}$

Reference

Lymph node

metastasis

\begin{tabular}{lllc} 
Negative, $\mathrm{n}=27$ & $17(63)$ & $10(37)$ & Reference \\
Positive, $\mathrm{n}=3$ & $2(66.7)$ & $1(33.3)$ & 0.90 \\
$\begin{array}{c}\text { Distant metastasis } \\
\text { Negative, } \mathrm{n}=26\end{array}$ & $17(65.4)$ & $9(36.6)$ & $\begin{array}{c}\text { Reference } \\
\text { Positive, } \mathrm{n}=4\end{array}$ \\
$2(50)$ & $2(50)$ & 0.57 \\
Overall survival & & & \\
Alive, $\mathrm{n}=22$ & $16(72.7)$ & $6(27.3)$ & Reference \\
Deceased, $\mathrm{n}=8$ & $3(37.5)$ & $5(62.5)$ & 0.08 \\
Recurrence & & & \\
No, $\mathrm{n}=21$ & $14(66.7)$ & $7(33.3)$ & Reference \\
Yes, $\mathrm{n}=9$ & $5(55.6)$ & $4(44.4)$ & 0.58 \\
\hline
\end{tabular}

${ }^{\mathrm{a}} \mathrm{P}<0.05$, indicates statistical significance. 'Reference' indicates that the correlation analysis of the classifications of clinical parameters were conducted in reference to the selected classification.

activity of the $\mathrm{Wnt} / \beta$-catenin signal transduction pathway. The cells were co-transfected with pRL-TK (Promega Corp., Madison, WI, USA) Renilla luciferase to normalize the trans- fection efficiency. The changes in the activity of TCF/LEF before and after transfection with DKK4 were detected by the analysis of luciferase activity.

RT-PCR analysis. Total mRNA was extracted from formalinfixed, paraffin-embedded human renal cancer tissues and matched adjacent normal tissues, as well as from RCC4(-) and T3-14(+) cells using TRIzol reagent(InvitrogenLife Technologies) in order for reverse transcription to be performed with the use of an RT reagent kit (Takara Bio, Inc., Shiga, Japan). The realtime experiments were conducted on an iQ5 Multicolor Real-Time PCR Detection System (Bio-Rad, Hercules, CA, USA) using the SYBR ${ }^{\circledR}$-Green real-time PCR kit (Takara Bio, Inc.). The PCR reactions consisted of $10 \mathrm{~min}$ at $95^{\circ} \mathrm{C}$ followed by 50 cycles at $95^{\circ} \mathrm{C}$ for $15 \mathrm{sec}$, at $60^{\circ} \mathrm{C}$ for $60 \mathrm{sec}$. The data were calculated using the $2^{-\Delta \Delta \mathrm{Ct}}$ method normalized to actin, the individual internal control. The primers used were as follows: DKK4 forward, 5'-TGGCACACATGCAGAA GGAACAAC-3' and reverse, 5'-AATGACGAGCACAGC AAAGTCCAG-3'; actin forward, 5'-ACCCAGCACAA TGAAGATCA-3' and reverse, 5'-CGATCCACACGGAG TACTTG-3'; cyclin D1 forward, 5'-AGAAGCTGTGCA TCTACACCGACA-3' and reverse, 5'-TGATCTGTTTGTT CTCCTCCGCCT-3'; c-myc forward, 5'-TGCAGCTGCTTA GACGCTGGATTT-3' and reverse, 5'-TGTTGGTGAAGCT AACGTTGAGGG-3'.

Western blot analysis. Nuclear protein and cytoplasmic protein were extracted separately with the use of western blotting luminol reagent, ProteoJET ${ }^{\mathrm{TM}}$ cytoplasmic and nuclear protein kits (Santa Cruz Biotechnology, Santa Cruz, CA, USA). Protein quantification was performed using the Coomassie brilliant blue G250 kit (Beyotime, Jiangsu, China). For western blot analysis, a total of $20 \mu \mathrm{g}$ of cell protein lysate was loaded per lane. Samples were resolved in $12 \%$ precise protein gels (Pierce, Courtaboeuf, France) and transferred onto polyvinylidene fluoride (PVDF) membranes (Amersham Biosciences, Fairfield, CT, USA). The membranes were immersed in a solution of $0.3 \%$ skim milk, Tris-buffered saline (TBS) and 0.1\% Tween-20 for $1 \mathrm{~h}$ and then probed with primary polyclonal and monoclonal antibodies against DKK4, actin, cyclin D1, c-myc and $\beta$-catenin (all purchased from Epitomics, Burlingame, CA, USA). The blots were washed in TBS and incubated with secondary antibodies. Proteins were enhanced by chemiluminescence (ECL, Santa Cruz) for visualization. The reported protein expression levels were expressed relative to the $\beta$-catenin levels.

Analysis of in vivo tumor growth. A total of 16 female nude mice (strain BALB/c nude; Shanghai experimental Animal Center, Chinese Academy of Sciences), aged 4 to 5 weeks received subcutaneous injections of empty vector-transfected A-498 cells (group a, $n=4$ ) or A-498 cells stably transfected with DKK4 (group b, n=4), and empty vector-transfected 786-O cells (group c, $n=4$ ) or 786-O cells stably transfected with DKK4 (group d, n=4), in the left armpit at a volume of $200 \mu$ l. Tumor size was measured using calipers once a week for 35 days, and was calculated on the basis of width (x) and length (y) according to the following formula: $x^{2} y / 2$, where $x<y$. After the mice were sacrificed, the tumors were resected and weighed. RNA and protein were extracted from the tumor 


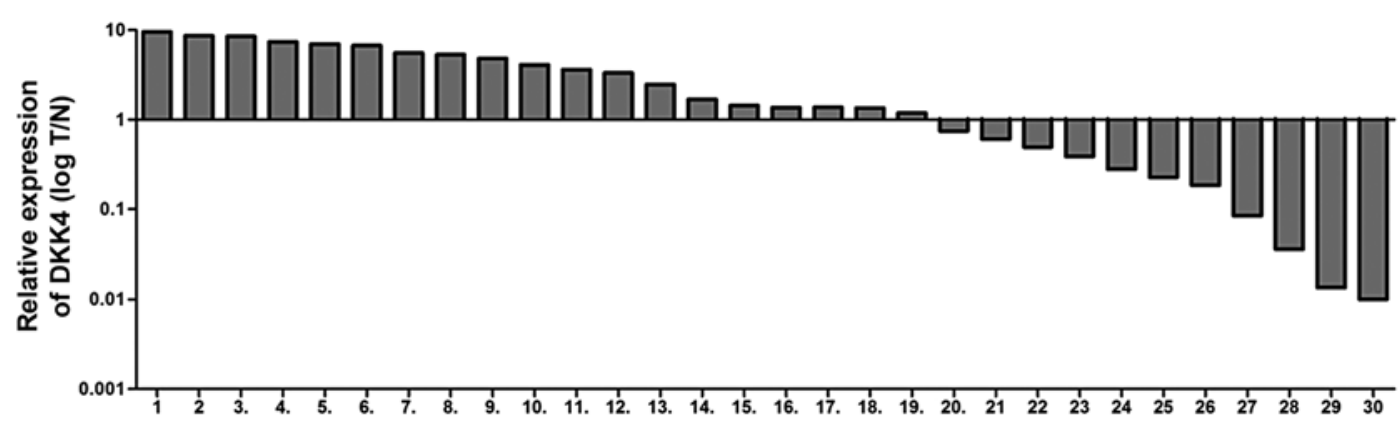

Figure 1. Dickkopf homolog 4 (DKK4) messenger RNA (mRNA) expression levels in original human renal tumor/adjacent lymph nodes (T/N), presented as $-\log (10)$ ratios.

tissues for the determination of the expression levels of DKK4 mRNA and protein.

Statistical analysis. All experiments were performed in triplicate and all observed differences between the treated and control cells were analyzed by the Student's t-test. The data are presented as the means \pm SD of 3 independent experiments. Correlation analyses were conducted using non-parametric tests and values of $\mathrm{p}<0.05$ were considered to indicate statistically significant differences.

\section{Results}

DKK4 mRNA expression levels in renal cancer tissues and adjacent normal tissues. RT-PCR was performed to examine the expression of DKK4 in renal cancer tissues and adjacent normal kidney tissues. As shown in Fig. 1, the mRNA expression levels of DKK4, in 30 paired tissues, were increased in 19 ccRCC tissues (63.3\%) compared to the adjacent tissues. The correlation between DKK4 expression levels and clinicophathological characteristics was also investigated, including gender, Fuhrman grade, pathological tumor classification (pT), pathological lymph node status $(\mathrm{pN})$, pathological metastasis status (pM) and outcomes (survival and recurrence). There was no significant association observed between DKK4 expression levels and clinicophathological parameters ( $\mathrm{p}>0.05)$ apart from the age of the patients (Table I).

Cell viability, invasion and apoptosis of cells with high DKK4 expression. Following transfection of the 786-O and A-498 cells with the pCDH-DKK4 expression plasmid, DKK4 expression levels were confirmed by qRT-PCR. DKK4 overexpression was observed in both the 786-O and A-498 cells (Fig. 2A). Analyses of cell viability (MTS) and cell invasion, as well as TCF/LEF reporter and apoptosis assays were performed using stably transfected 786-O and A-498 cells with high expression levels of DKK4. The enhanced growth of the transfected 786-O and A-768 cells was observed by MTS assay (Fig. 2B). DKK4 promoted the in vitro invasion ability of the 786-O and A-768 cells (Fig. 2C). The relative TCF/LEF activity was significantly decreased in the 786-O and A-768 cells co-transfected with the TOPFlash reporter plasmid compared with the controls (Fig. 2D). No significant differences were observed between the 2 cell lines and the control cells as regards the number of apoptotic cells (Fig. 2E).
In vivo experiments. Tumors developed in all the mice. Tumor volumes in the mice overexpressing DKK4 were larger at 2 weeks, and significantly larger at 6 weeks than those of the control mice at the respective time points $(\mathrm{p}<0.05$, Fig. 3A). The final tumor weights of groups $a, b, c$ and $d$ were $2.173 \pm 0.097,3.014 \pm 0.201,2.51 \pm 0.217$ and $3.673 \pm 0.142 \mathrm{~g}$, respectively. The results from qRT-PCR (Fig. 3B) and western blot analysis (Fig. 3C) confirmed the overexpression of DKK4 in the tumors formed from 786-O and A-498 transplanted cells transfected with pCDH-DKK4 compared with those formed from cells transfected with the empty vector $(\mathrm{p}<0.001)$.

VHL protein expression and DKK4 expression in ccRCC tissues. VHL protein expression in another $50 \mathrm{ccRcc}$ tissues was detected by western blot analysis. VHL protein was negatively expressed in $60 \%$ of the tissues (30/50) which were classified as the VHL(-) group, while the remaining 20 tissues were classified as the VHL(+) group. DKK4 expression levels in all 50 tissues was detected by qRT-PCR, and DKK4 was overexpressed in $68 \%(34 / 50)$ of the tissues. The DKK4 overexpression ratio in the VHL(-) group was higher than that in the VHL(+) group (50\% vs. $18 \%$ ), and the difference was statistically significant $(\mathrm{p}<0.05)$. In addition, the overexpression of DKK4 correlated with the expression of the VHL gene $(\mathrm{r}=0.403, \mathrm{p}<0.05)$. DKK4 overexpression also correlated with VHL gene expression $(r=0.403, p<0.05)$.

Detection of $\beta$-catenin, cyclin DI and c-myc expression. The protein expression levels of $\beta$-catenin in the cytoplasm and nucleus were compared between the tumor and normal tissues. The expression levels of $\beta$-catenin in the nucleus of the tumor cells were decreased compared with those in the nucleus of normal cells; however, the differences in the expression levels in the cytoplasm of tumor and normal tissues were not statistically significant (Fig. 4A). Thus, the $\beta$-catenin expression levels of the downstream genes, cyclin D1 and c-myc, were examined in the VHL(+) and VHL(-) groups by qRT-PCR. The decreased expression levels of these genes in the VHL(-) tissues were also found to be statistically significant (Fig. 4B, p<0.05). The expression levels of phosphorylated $\beta$-catenin ( $p-\beta$-catenin) presented a sharp increase in the VHL(+) tissues; however, there was no notable difference observed between the total $\beta$-catenin expression levels in the VHL(+) and VHL(-) tissues (Fig. 4C). DKK4, cyclin D1, c-myc and $\beta$-catenin expression levels were also detected in RCC4(-) and T3-14(+) cells by 

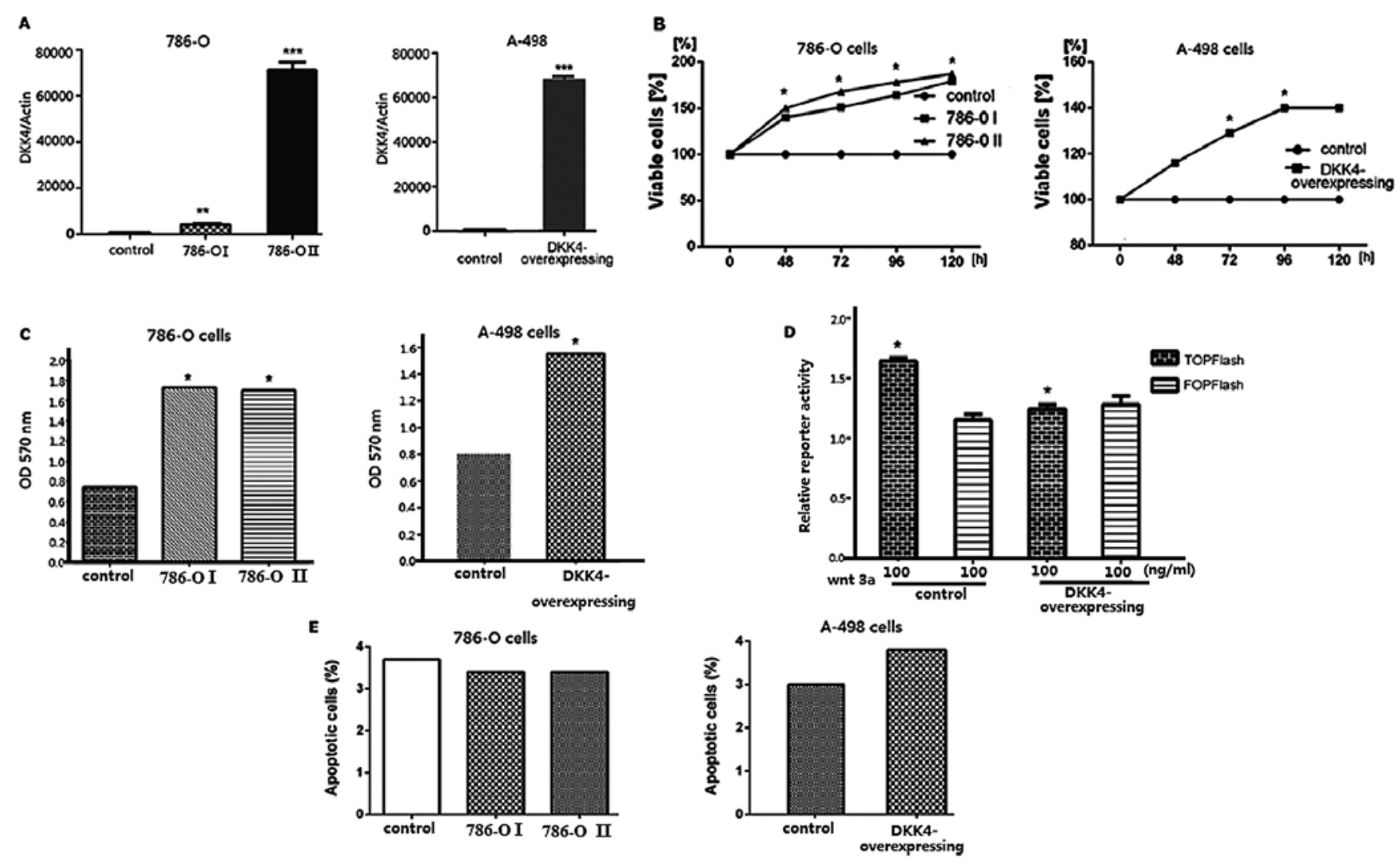

Figure 2. (A) Establishment of Wnt antagonist dickkopf homolog 4 (DKK4) transfectants. DKK4 expression levels were analyzed in stable transfectants by qRT-PCR. (B) Cell viability (MTS) assay in the control (transfected with empty plasmid) and DKK4-transfected cells. (C) Cell invasion assay in control and DKK4-transfected cells. (D) A TCF/LEF receptor assay. TOPFlash indicates a TCF-receptor plasmid that contains a wild-type T-cell factor (TCF) binding site; FOPFlash, TCF-receptor plasmid that contained a mutant-type TCF binding site. (E) Apoptosis assay on transfected 786-O and A-498 cells. ${ }^{*} \mathrm{p}<0.05,{ }^{* *} \mathrm{p}<0.01$, *** $\mathrm{p}<0.001$ compared with controls (empty vector transfectants).
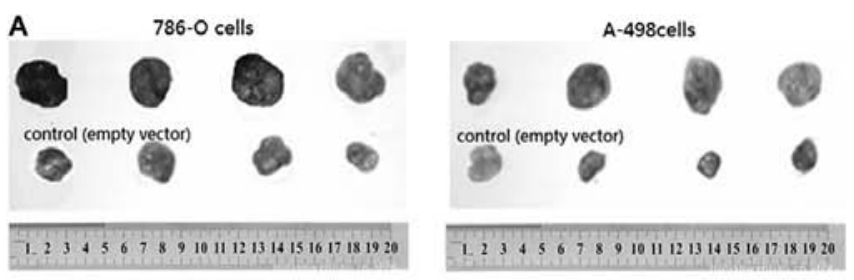

C

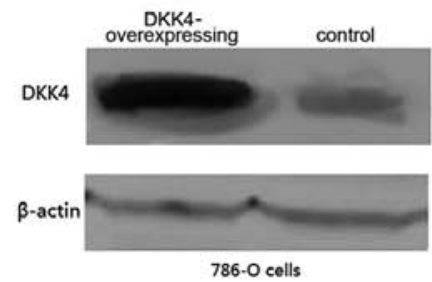

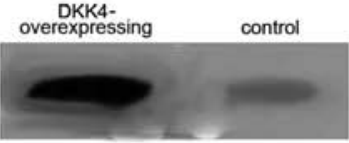

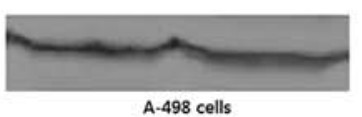

B

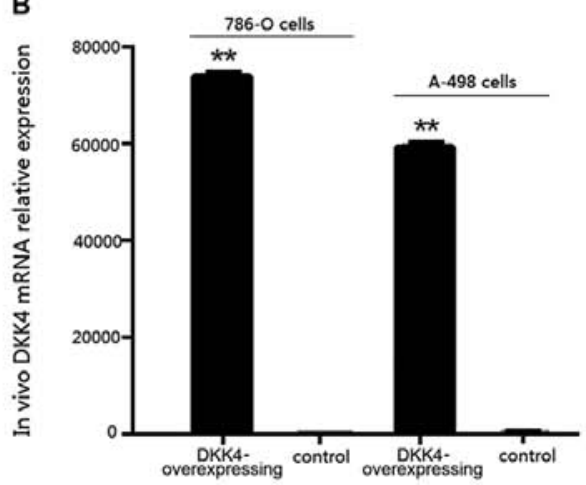

Figure 3. In vivo experiments with empty and with dickkopf homolog 4 (DKK4)-transfected renal cancer cells. (A) Determination of tumor volume in nude mice implanted with 786-O and A-498 cells transfected with empty vector (control) or with cells transfected with pCDH-DKK4 plasmid. (B) Detection of DKK4 mRNA expression levels in mice implanted with DKK4-overexpressing cells or empty vector-transfected control cells. (C) Detection of DKK4 protein expression levels in mice implanted with DKK4-overexpressing cells or empty vector-transfected control cells. ${ }^{* *}$ p $<0.01$ compared with controls (empty vector transfectants).

qRT-PCR and western blot analysis. The DKK4 expression levels in the RCC4(-) cells were 67.555-fold higher compared with those in the T3-14(+) cells, while the cyclin D1 and c-myc expression levels were markedly decreased in the RCC4(-) cells (Fig. 4D). In addition we also determined the cyclin D1 and c-myc expression levels in human embryonic kidney (HEK)293 cells transfected with the plasmid vector, pCDHDKK4. DKK4 was highly expressed in the HEK293 cells 
A
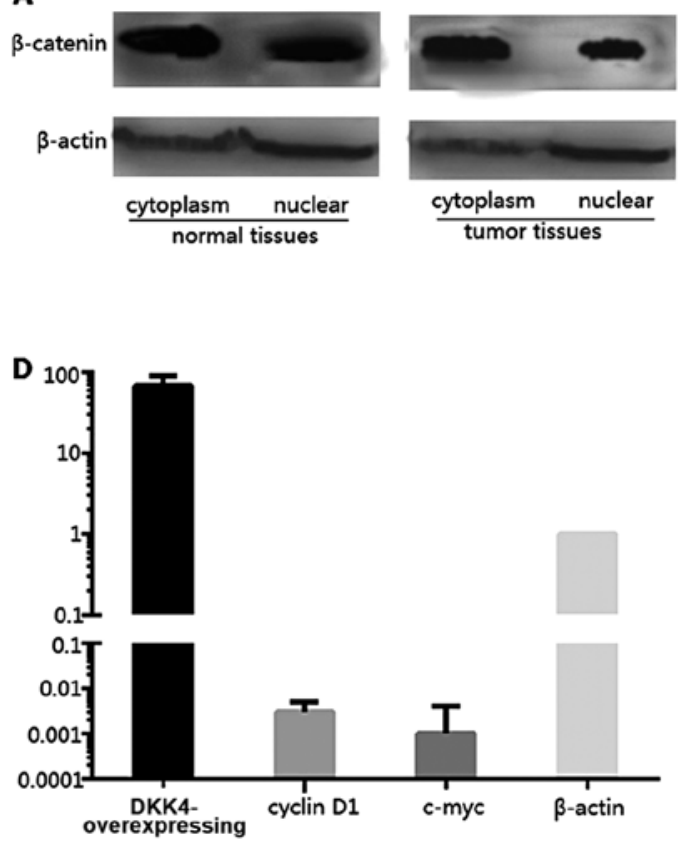

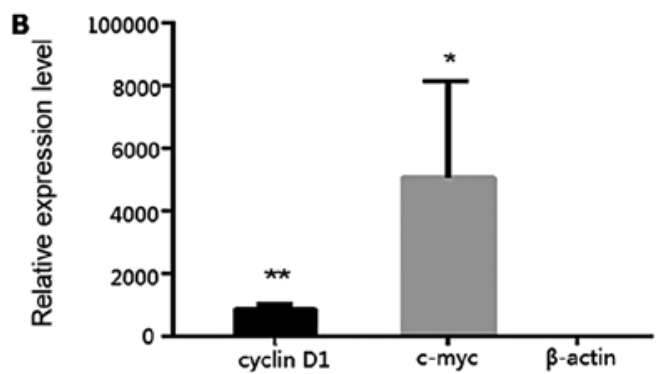

E

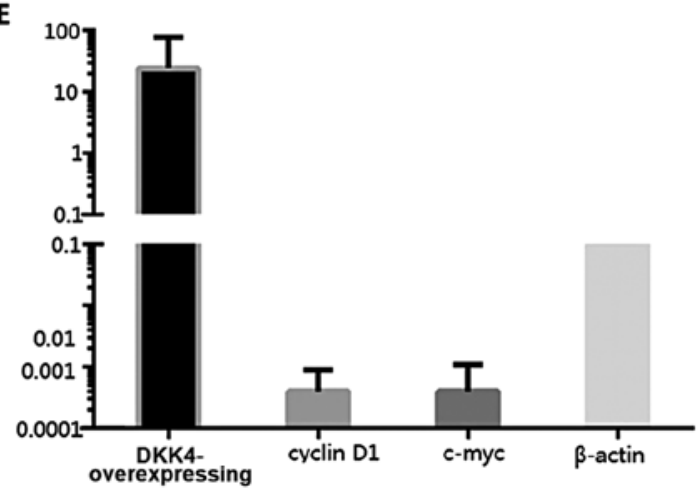

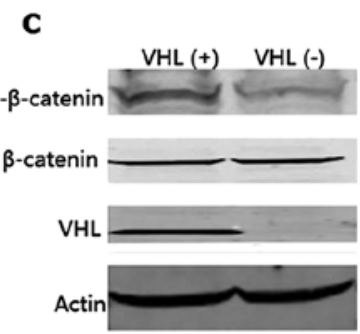

$\mathbf{F}$

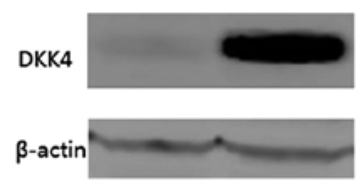

Figure 4. (A) Western blot analysis of $\beta$-catenin cytoplasmic and nuclear expression levels. (B) Relative expression levels of $\beta$-catenin downstream target genes, cyclin D1 and c-myc, were decreased significantly in Von Hippel-Lindau negative tissues [VHL(-)] (p<0.05). (C) Expression levels of VHL protein, $\beta$-catenin and phosphorylated $\beta$-catenin in RCC4(-) and T3-14(+) cells. (D) Expression levels of DKK4, cyclin D1, c-myc in RCC4 (-) and T3-14 (+) cells. (E) Expression levels of DKK4, cyclin D1, c-myc in pCDH-DKK4 plasmid-transfected human embryonic kidney (HEK)293 cells. (F) Western blot analysis of DKK4 expression levels in HEK293 cells.

(Fig. 4F), and the expression levels of cyclin D1 and c-myc were significantly decreased (Fig. 4E).

\section{Discussion}

DKK4 expression was determined in 30 cases of ccRCC, and it was found to be highly increased in $63.3 \%$ of the ccRCC tissues compared with the adjacent normal tissues (19/30). No correlation was observed between DKK4 expression and clinicopathological parameters, such as Fuhrman grade, pathological stage, lymph node and distant metastasis, survival and recurrence. The overexpression of DKK4 indicated that its activation is involved in ccRCC. In 1999 it was reported that DKK4 regulates the Wnt/ $\beta$-catenin signaling pathway negatively (26), but since then, few studies have investigated the effects of DKK4 on tumorigenesis and the devolopment of ccRCC. In the current study, cells that expressed high levels of DKK4 were found to have enhanced cell viability and a greater invasion potential. Matsui et al (24) reported that DKK2 and DKK4 were upregulated in colorectal cancer, and that DKK4 expression levels correlated with nuclear $\beta$-catenin levels. However, Fatima et al (25), demonstrated, with functional assays, that DKK4 overexpression inhibited cell proliferation, reduced colony formation and suppressed cell migration in hepatocellular carcinoma. The DKK family is a family of secretory glycoproteins, consisting of two structuraly conserved cysteine-rich domains and each domain contains 10 cysteine residues (26). The amino-terminal domain (Cyst-1) is domain-specific, and the carboxy-terminal domain (Cyst-2) is highly conserved. Cysteine residues on Cyst-2 have the typical cysteine pattern of the colipase domain and can therefore combine colipase tightly, which participates in fat hydrolysis (27). Thus, the structure of the DKK family may be associated with the Wnt/ $\beta$-catenin signaling pathway (28). By detecting cells transfected with LEF- 1 and $\beta$-catenin, DKK4 is selected as a potential target gene of the non-canonical Wnt signaling pathway (Wnt/PCP), and also participates in cell movements during the morphogenesis period (29). In fact, apart from the Wnt/ $\beta$-catenin pathway, Wnt can be activated by other pathways dependent on Jun N-terminal kinase (JNK). JNK activation induces apoptosis, and JNK activation by DKK 3 has been reported to be involved in the Wnt pathway regulation in prostate and breast cancer $(30,31)$. However, the effects of DKK4 on the Wnt/JNK pathway in ccRCC and the coresponding mechanisms require further verification.

The results of the current study demonstrated that the canonical Wnt/ $\beta$-catenin was inhibited, leading to the reduced expression of $\beta$-catenin and its downstream genes, cyclin D1 and c-myc, as well as a decrease in TCF/LEF activity. However, in the study by Fatima et al (25), the expression levels of DKK4 $\beta$-catenin and cyclin D1 were decreased in hepatoma carcinoma cells, and $\beta$-catenin was accumulated in the cytoplasm. Similar results have been observed in colorectal cancer (32). Under the activation of Wnt/ $\beta$-catenin signaling pathway, DKK4 overexpression can inhibit the activities of $\beta$-catenin/TCF- 4 effectively, as well as cell cycle proliferation and conversion. DKK4 may play different roles in tumorigenesis and in the development of different types of tumor. Further studies are required in order to elucidate the underlying mechanisms.

In vivo studies offer a more realistic microenvironment for the growth of tumor cells. In this study, cells overexpressing DKK4 were implanted into BALB/c nude mice, in order to investigate the effects of DKK4 activation on the oncogenicity 
of ccRCC. The neoplasm incidence rate was $100 \%$, and the tumor weights of the mice implanted with cells overexpressing DKK4 were significantly higher than those of the control mice implanted with an empty vector. DKK4 is considered to inhibit the development of tumors, as an antagonist of the Wnt pathway and has also been reported to be able to inhibit the proliferation of tumor cells in hepatic carcinomas (25). However, the issue of whether DKK4 acts as a tumor inhibitor or promoter remains to be resolved.

VHL is believed to induce the oxygen-deficient environment in cells, as it activates many HIF-mediated signaling pathways $(11,33)$. The abnormal expression of VHL promotes the migration of $\beta$-catenin from the cytoplasm into the nucleus, as well as its combination with TCF and LEF, and the activation of cyclin D1 and c-myc, and consequently tumorigenesis $(19,34)$. The combination of $\beta$-catenin with TCF/LEF in the nucleus activates downstream genes (35). In our study, in a total of 50 cases of ccRCC, in the VHL(-) group, DKK4 was found to be overexpressed in $50 \%$ of the specimens, contrary to the $\mathrm{VHL}(+)$ group, where only an $18 \%$ rate presented a significant difference $(\mathrm{p}<0.05)$. The high expression of DKK4 correlated with the expression of VHL $(r=0.403 ; p<0.05)$. As a positive cell cycle factor, cyclin D1 is associated with uncontrollable cell proliferation, as a tumorigenesis factor (36). The overexpression of cyclin D1 induces system disorders in the normal cell cycle (37). When c-myc, another well known nuclear oncogene, is regulated by carcinogenic factors, it escapes the normal regulatory cell cycle check, leading to its continuous overexpression and resulting in the alteration of normal cells ubto tumor cells (38). Thus, the abnormal expression of c-myc may be a basis for tumorigenesis.

Recent studies have been conducted on miRNAs in ccRCC. It has been reported that miR-1826 is upregulated in $\mathrm{VHL}(+)$ ccRCC cells compared with VHL-inactivated 786-O and A-498 cell lines. miR-1826 has also been found responsible for the decreased expression levels of $\beta$-catenin and the survival of its downstream genes (39). VHL expression can also be inhibited by miR-23, but $\beta$-catenin/TCF- 4 transcription activity, as well as the proliferation and invasion of tumor cells can be enhanced. Nevertheless, once the VHL gene is transduced all effects are abolished (40). The oncogenic activity of miRNAs related or not with high expression levels of DKK4, as well as the effects of VHL on DKK4 require further investigation.

DKK4 blocks the canonical $\mathrm{Wnt} / \beta$-catenin pathway in ccRCC; however, its effects on the biological behavior of ccRCC may also be due to other activated pathways. Further studies are required to fully elucidate the roles of DKK4 in ccRCC tumorigenesis and tumor progression.

\section{References}

1. Ljungberg B, Hanbury DC, Kuczyk MA, et al: Renal cell carcinoma guideline. Eur Urol 51: 1502-1510, 2007.

2. Zhang Z, Wondergem B and Dykema K: A comprehensive study of progressive cytogenetic alterations in clear cell renal cell carcinoma and a new model for ccRCC tumorigenesis and progression. Adv Bioinformatics 2010: 428325, 2010.

3. Lam JS, Leppert JT, Figlin RA and Belldegrun AS: Surveillance following radical or partial nephrectomy for renal cell carcinoma. Curr Urol Rep 6: 7-18, 2005.

4. Huang Y, Dai Y, Yang J, et al: Microarray analysis of microRNA expression in renal clear cell carcinoma. Eur J Surg Oncol 35: $1119-1123,2009$.
5. Figlin RA, Pierce WC, Kaboo R, et al: Treatment of metastatic renal cell carcinoma with nephrectomy, interleukin-2 and cytokine-primed or CD8 (+) selected tumor infiltrating lymphocytes from primary tumor. J Urol 158: 740-745, 1997.

6. Nelson WJ and Nusse R: Convergence of Wnt, $\beta$-catenin, and cadherin pathways. Science 303: 1483-1487, 2004.

7. Capurro MI, Xiang YY, Lobe C and Filmus J: Glypican-3 promotes the growth of hepatocellular carcinoma by stimulating canonical Wnt signaling. Cancer Res 65: 6245-6254, 2005.

8. Rask K, Nilsson A, Brännström M, et al: Wnt-signalling pathway in ovarian epithelial tumours: increased expression of $\beta$-catenin and GSK3 $\beta$. Br J Cancer 89: 1298-1304, 2003.

9. Chien AJ, Moore EC, Lonsdorf AS, et al: Activated Wnt/ $\beta$-catenin signaling in melanoma is associated with decreased proliferation in patient tumors and a murine melanoma model. Proc Natl Acad Sci USA 106: 1193-1198, 2009.

10. Gilbertson RJ: Medulloblastoma: signalling a change in treatment. Lancet Oncol 5: 209-218, 2004.

11. Banumathy $\mathrm{G}$ and Cairns P: Signaling pathways in renal cell carcinoma. Cancer Biol Ther 10: 658-664, 2010.

12. Logan CY and Nusse R: The Wnt signaling pathway in development and disease. Annu Rev Cell Dev Biol 20: 781-810, 2004.

13. Fang D, Hawke D, Zheng Y, et al: Phosphorylation of $\beta$-catenin by AKT promotes $\beta$-catenin transcriptional activity. J Biol Chem 282: 11221-11229, 2007.

14. Jho EH, Zhang T, Domon C, Joo CK, Freund JN and Costantini F: Wnt $/ \beta$-catenin/Tcf signaling induces the transcription of Axin2, a negative regulator of the signaling pathway. Mol Cell Biol 22: 1172-1183, 2002

15. Hirata H, Hinoda Y, Majid S, et al: DICKKOPF-4 activates the noncanonical c-Jun-NH2 kinase signaling pathway while inhibiting the Wnt-canonical pathway in human renal cell carcinoma. Cancer 117: 1649-1660, 2011.

16. Pfaff E, Becker S, Günther A and Königshoff M: Dickkopf proteins influence lung epithelial cell proliferation in idiopathic pulmonary fibrosis. Eur Respir J 37: 79-87, 2011.

17. Pendás-Franco N, García J, Peña C, et al: DICKKOPF-4 is induced by $\mathrm{TCF} / \beta$-catenin and upregulated in human colon cancer, promotes tumour cell invasion and angiogenesis and is repressed by $1 \alpha, 25$-dihydroxyvitamin D3. Oncogene 27 : 4467-4477, 2008.

18. Katoh Y and Katoh M: Comparative genomics on DKK2 and DKK4 orthologs. Int J Mol Med 16: 477-481, 2005.

19. Peruzzi B, Athauda G and Bottaro DP: The von Hippel-Lindau tumor suppressor gene product represses oncogenic $\beta$-catenin signaling in renal carcinoma cells. Proc Natl Acad Sci USA 103: 14531-14536, 2006

20. Kim YS, Kang YK, Kim JB, Han S, Kim KI and Paik SR: $\beta$-catenin expression and mutational analysis in renal cell carcinomas. Pathol Int 50: 725-730, 2000.

21. Tanimoto K, Makino Y, Pereira T and Poellinger L: Mechanism of regulation of the hypoxia-inducible factor- $1 \alpha$ by the von Hippel-Lindau tumor suppressor protein. EMBO J 19: 4298-4309, 2000.

22. Ma X, Yang K, Lindblad P, Egevad L and Hemminki K: VHL gene alterations in renal cell carcinoma patients: novel hotspot or founder mutations and linkage disequilibrium. Oncogene 20: 5393-5400, 2001.

23. Patel PH, Chadalavada RS, Chaganti R and Motzer RJ: Targeting von Hippel-Lindau pathway in renal cell carcinoma. Clin Cancer Res 12: 7215-7220, 2006

24. Matsui A, Yamaguchi T, Maekawa S, et al: DICKKOPF-4 and -2 genes are upregulated in human colorectal cancer. Cancer Sci 100: 1923-1930, 2009.

25. Fatima S, Lee N, Tsang F, et al:: Dickkopf 4 (DKK4) acts on Wnt $/ \beta$-catenin pathway by influencing $\beta$-catenin in hepatocellular carcinoma. Oncogene 31: 4233-4244, 2012.

26. Krupnik VE, Sharp JD, Jiang C, et al: Functional and structural diversity of the human Dickkopf gene family. Gene 238: 301-313, 1999.

27. Aravind L and Koonin EV: A colipase fold in the carboxyterminal domain of the Wnt antagonists-the Dickkopfs. Curr Biol 8: R477-R478, 1998.

28. van Tilbeurgh H, Bezzine S, Cambillau C, Verger R and Carrière F: Colipase: structure and interaction with pancreatic lipase. Biochim Biophys Acta 1441: 173-184, 1999.

29. Bazzi H, Fantauzzo KA, Richardson GD, Jahoda CA and Christiano AM: The Wnt inhibitor, Dickkopf 4, is induced by canonical Wnt signaling during ectodermal appendage morphogenesis. Dev Biol 305: 498-507, 2007. 
30. Veeck J, Bektas N, Hartmann A, et al: Wnt signalling in human breast cancer: expression of the putative Wnt inhibitor Dickkopf-3 (DKK3) is frequently suppressed by promoter hypermethylation in mammary tumours. Breast Cancer Res 10: R82, 2008.

31. Zenzmaier C, Untergasser G, Hermann M, Dirnhofer S, Sampson N and Berger P: Dysregulation of Dkk-3 expression in benign and malignant prostatic tissue. Prostate 68: 540-547, 2008.

32. Baehs S, Herbst A, Thieme SE, et al: Dickkopf-4 is frequently down-regulated and inhibits growth of colorectal cancer cells. Cancer Lett 276: 152-159, 2009.

33. Morais C, Johnson DW, Gobe G: The VHL-HIF signaling in renal cell carcinoma: promises and pitfalls. In: Emerging Research and Treatments in Renal Cell Carcinoma. Amato RJ (ed). InTech Publishers, Croatia, pp57-82, 2011.

34. Linehan WM, Rubin JS and Bottaro DP: VHL loss of function and its impact on oncogenic signaling networks in clear cell renal cell carcinoma. Int J Biochem Cell Biol 41: 753-756, 2009.

35. Mao B, Wu W, Davidson G, et al: Kremen proteins are Dickkopf receptors that regulate Wnt/beta-catenin signalling. Nature 417: 664-667, 2002.
36. Baldin V, Lukas J, Marcote M, Pagano M and Draetta G: Cyclin D1 is a nuclear protein required for cell cycle progression in G1. Genes Dev 7: 812-821, 1993.

37. Wu JF, Shao JC, Wang DB, Qin R and Zhang H: Expression and significance of cell cycle regulators in gastric carcinoma. Ai Zheng 24: 175-179, 2005.

38. Spencer CA and Groudine M: Control of c-myc regulation in normal and neoplastic cells. Adv Cancer Res 56: 1-48, 1991.

39. Hirata H, Hinoda Y, Ueno K, Nakajima K, Ishii N and Dahiya R: MicroRNA-1826 directly targets $\beta$-catenin (CTNNB1) and MEK1 (MAP2K1) in VHL-inactivated renal cancer. Carcinogenesis 33: 501-508, 2012.

40. Chen L, Han L, Zhang K, et al: VHL regulates the effects of miR-23b on glioma survival and invasion via suppression of HIF-1 $\alpha /$ VEGF and $\beta$-catenin/Tcf-4 signaling. Neuro Oncol 14: 1026-1036, 2012. 